\title{
Gyrokinetic nonlinear isotope effects in tokamak plasmas
}

\author{
J. Garcia ${ }^{1}$, T. Görler ${ }^{2}$, F. Jenko ${ }^{3}$ G. Giruzzi ${ }^{1}$ \\ ${ }^{1}$ CEA, IRFM, F-13108 Saint-Paul-lez-Durance, France. \\ ${ }^{2}$ Max Planck Institute for Plasma Physics, Boltzmannstr. 2, 85748 Garching, Germany \\ ${ }^{3}$ Department of Physics and Astronomy, University of California, Los Angeles, California 90095, USA
}

PACS numbers: 52.25.Fi

Previous observations of an isotope dependent energy confinement in magnetically confined plasmas are still largely unexplained and its interaction with apparently unrelated plasma conditions has become one of the main unsolved issues with great relevance for nuclear fusion. By means of numerical studies based on the gyrokinetic theory, we quantitatively show how the plasma microturbulence can depend on the isotope mass through nonlinear multiscale effects, involving the interplay and competition between zonal flow shearing, electromagnetic effects and the torque applied. This observation can help to disentangle some of the pieces that form the puzzle of the isotope effect and to provide useful guidelines for establishing priorities in future DT campaigns.

The thermal energy confinement time has been observed to vary significantly with the mass of the main isotope in broad experimental conditions performed in different tokamaks [1]. Of particular interest is the isotope exchange between the fusion of deuterium (DD) and deuterium-tritium (DT) nuclei, as the first reaction is the one usually produced in present day tokamaks whereas the second one produces much higher rates of fusion energy and it is envisaged to generate fusion power in future tokamaks as ITER. In specific experiments performed in the 1990's both in the Tokamak Fusion Test Reactor (TFTR) and the Joint European Torus (JET) a broad and unexpected variety of results, including strong ion heat transport reductions, were obtained when exchanging the main ion species in the plasma composition from Deuterium (D) to a mixture of Deuterium-Tritium (DT) [2,3]. This is in contradiction with the heat flux scaling expected from the so-called Gyro-Bohm estimate for turbulent transport which assumes that the nature of the heat transport is basically local and that the characteristic step size of collisional transport and turbulent structures both increase with the plasma gyroradius, leading to a mass dependence of the ion heat flux $Q_{i} m_{i}^{1 / 2}$. These inconsistencies were labelled as the isotope effect. Several physical mechanisms have been proposed as explanations, such as the impact of zonal flows [4] or the change of the edge plasma conditions [5], however a full explanation of the isotope effects on plasma confinement has been elusive, in particular its dependence on the external input heating power [2] or the increasing of the correlation length when the heat decreases in dedicated recent isotope experiments in Asdex-U [6] are still unexplained.

In this letter, the gyrokinetic theory is used in order to quantify the implications a DD to DT change could have in burning plasmas as well as providing useful guidelines for establishing scientific priorities in future DT campaigns like the one expected at JET and ITER [7] with the aim of disentangling some of the pieces forming the isotope effect. Since the analysis of previous DT results is challenging given the fact that the data quality is not comparable to present-day standards and crucial information as the $\mathrm{D}$ and $\mathrm{T}$ densities are not available [8], we perform gyrokinetic simulations 
based on an established ITER modeling scenario obtained from a JET extrapolation. It corresponds to a so called hybrid regime with high input power and $\beta$, with $\beta=2 \mu_{0}<P>i B^{2}$, where $\mathrm{P}$ is the plasmas pressure and B the magnetic field. A summary of the main plasma parameters can be found in [9] together with the plasma equilibrium details. In this study, a mixture of $50 \% \mathrm{D}$ and $50 \% \mathrm{~T}$ has been assumed.

The gyrokinetic code GENE [10] has been used for computing linear and nonlinear microturbulence characteristics in the core plasma region. All simulations are local and they included kinetic electrons, collisions and electromagnetic effects. The code employs field aligned coordinates, where $\mathrm{x}$ is the radial coordinate, $\mathrm{z}$ is the coordinate parallel along the field, and $\mathrm{y}$ is the binormal coordinate. Grid parameters were as follows: perpendicular box sizes $[\mathrm{Lx}, \mathrm{Ly}]=[153,125]$ in units of the ion Larmor radii, perpendicular grid discretization $[\mathrm{nx}, \mathrm{nky}]=[192,32], 32$ point discretization in the parallel direction, 48 points in the parallel velocity direction, and 12 magnetic moments. The instability linear growth rate $\gamma$ and the external ExB flow shear rate, $\gamma_{E x B, \text { ext }}=r / q d \Omega / d r$, with $\Omega$ the toroidal angular velocity, are in units of $\mathrm{C}_{\mathrm{s}} / \mathrm{R}$, with $C_{s}=\sqrt{T_{e} / m_{D}}$ and $\mathrm{T}_{\mathrm{e}}$ the electron temperature, $\mathrm{m}_{\mathrm{D}}$ the deuterium mass and $\mathrm{R}$ the tokamak major radius. Both $\delta \mathrm{B}_{\perp}$ and $\delta \mathrm{B}_{\|}$fluctuations were computed. $\delta \mathrm{B}_{\|}$ cannot be neglected as it is important for setting the strength of electromagnetic coupling [11]. As representative of the inner core region, the dimensionless parameters of the discharge at $\rho=0.33$, with $\rho=\sqrt{\varphi}$ and $\varphi$ the normalized toroidal flux, fed into the gyrokinetic calculations are summarized in Table I.

\begin{tabular}{|c|c|c|c|c|c|}
\hline $\mathrm{s}$ & $\mathrm{q}$ & $\mathrm{T}_{\mathrm{e}} / \mathrm{T}_{\mathrm{i}}$ & $\mathrm{R} / \mathrm{L}_{\mathrm{Ti}}$ & $\mathrm{R} / \mathrm{L}_{\mathrm{Te}}$ & $\mathrm{R} / \mathrm{L}_{\mathrm{Ne}}$ \\
\hline 0.24 & 1.17 & 1.09 & 4.5 & 2.9 & 1.9 \\
\hline
\end{tabular}

TABLE I. Discharge dimensionless parameters at $\rho=0.33$ used as input in simulations. $s$ is the magnetic shear, $\mathrm{q}$ the safety factor, Te and Ti the electron and ion temperatures respectively, $\mathrm{R} / \mathrm{L}_{\mathrm{Ti}}=-R d T_{i} / d \rho / T_{i} \quad, \mathrm{R} / \mathrm{L}_{\mathrm{Te}}=$

$$
-R d T_{e} / d \rho / T_{e} \text { and } \mathrm{R} / \mathrm{L}_{\mathrm{Ne}}=\quad-R d N_{e} / d \rho / N_{e}
$$

At first, a linear analysis has been carried out for different plasma configurations. In figure 1a and 1b, the linear growth rates, $\gamma$, and frequencies, $\omega$, are shown. Over a wide normalized wavenumber range, $k_{y}=\hat{k} \quad{ }_{y} \rho_{s}$ where $\rho_{s}$ is the ion gyroradius with respect to the sound speed, Ion Temperature Gradient (ITG) modes are unstable. The maximum growth rate, $\gamma_{\max }$, is lower for DT, $\gamma_{\max , D T}=0.034\left[C_{S} / R\right]$, than for DD, $\gamma_{\max , D D}=0.042\left[C_{S} / R\right]$, however this trend closely follows the Gyro-Bohm scaling as $\gamma_{\max , D T} \gamma_{\max , D D} \sqrt{m_{D D} / m_{D T}}$. The influence of electromagnetic effects was studied performing the same type of simulation by reducing the electron beta $\beta_{e}=2 \mu_{0}<P_{e}>\dot{i} B^{2}$ from the nominal value $1.2 \%$ to $1.2 \times 10^{-2} \%$ (i.e. performing an electrostatic 
simulation). The growth rates increase for both DD and DT, however their relative difference remains similar to the electromagnetic case. Therefore, from the linear analysis, no significant deviation from the expected Gyro-Bohm scaling is obtained.
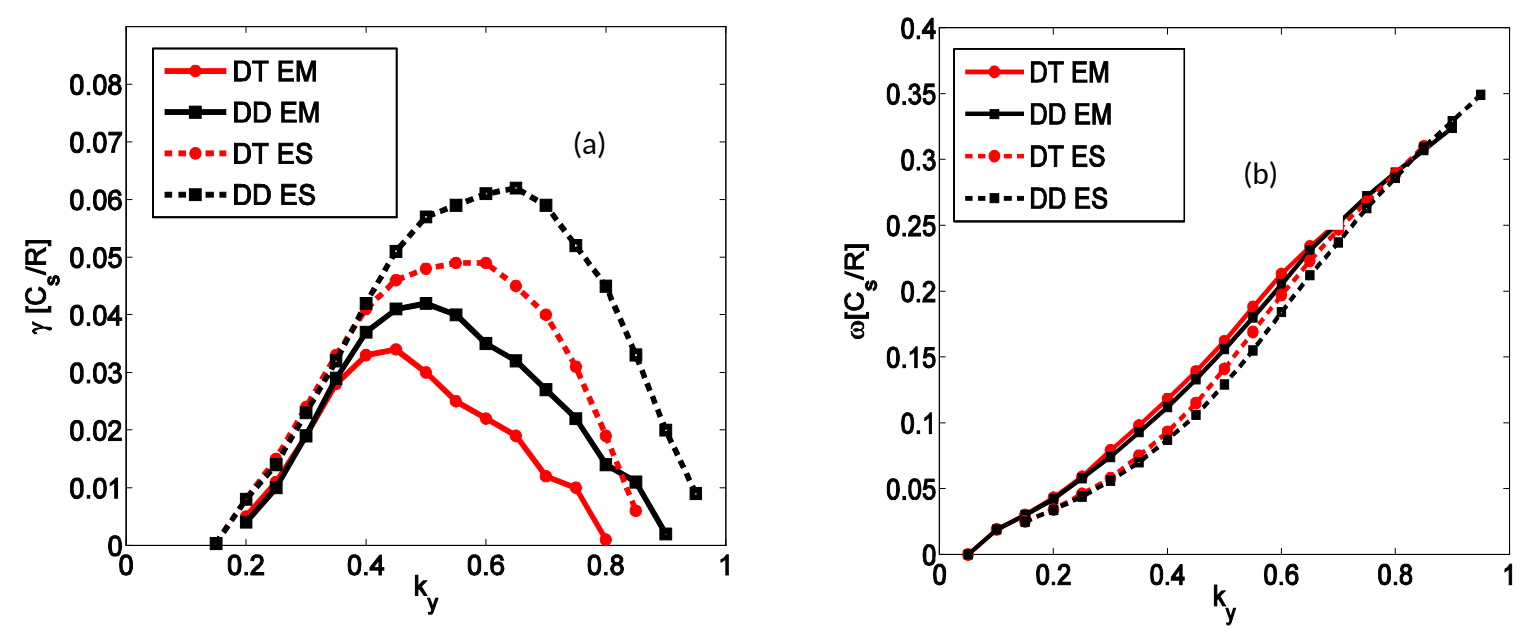

Figure 1 (a) Linear growth rates for DD and DT including electromagnetic effects (EM) or performing just a electrostatic (ES) simulations (b) Frequency for the previous cases.

\begin{tabular}{|c|c|c|c|c|c|c|c|c|c|c|}
\hline \# & \multicolumn{4}{|c|}{ Case } & $\mathrm{Q}_{\mathrm{i}}\left(\mathrm{kW} / \mathrm{m}^{2}\right)$ & $\gamma_{\max }[\mathrm{Cs} / \mathrm{R}]$ & $\mathrm{k}_{\mathrm{y} \text {, max }}$ & $\left|\gamma / k_{y}^{2}\right|_{m a}$ & $\gamma_{\text {ExB,zonal }}[\mathrm{Cs} / \mathrm{R}]$ & $\gamma_{\text {ExB,zonal }} / \gamma_{\max }$ \\
\hline 1 & DT & EM & ExB & $\mathrm{pfs}$ & 154 & & & & 0.37 & \\
\hline 2 & DD & EM & ExB & $\mathrm{pfs}$ & 271 & & & & 0.42 & \\
\hline 3 & DT & EM & & & 308 & 0.034 & 0.45 & 0.228 & 0.43 & 12.65 \\
\hline 4 & DD & EM & & & 363 & 0.042 & 0.50 & 0.238 & 0.45 & 10.71 \\
\hline 5 & DT & ES & ExB & $\mathrm{pfs}$ & 1270 & & & & 0.72 & \\
\hline 6 & $\mathrm{DD}$ & $\mathrm{ES}$ & ExB & $\mathrm{pfs}$ & 1505 & & & & 0.72 & \\
\hline 7 & DT & ES & & & 1491 & 0.050 & 0.60 & 0.269 & 0.69 & 14.08 \\
\hline 8 & DD & ES & & & 1366 & 0.062 & 0.65 & 0.262 & 0.68 & 10.48 \\
\hline 9 & DT & ES & ExB & & 1260 & & & & 0.72 & \\
\hline $\begin{array}{l}1 \\
0\end{array}$ & $\mathrm{DD}$ & ES & ExB & & 1303 & & & & 0.71 & \\
\hline
\end{tabular}

TABLE II. Results obtained for the different cases considered in this letter. $\gamma_{\max }$ and $\mathrm{k}_{\mathrm{y}, \max }$ have been calculated only for the cases with no impact of ExB flow shear

Non-linear simulations have been also performed for the previous linear runs, including as well the effect of the external ExB flow shear, $\gamma_{\mathrm{ExB}, \mathrm{ext}}$, which is known to quench turbulence in the ITG domain and the consequent parallel flow shear (pfs) which can destabilize it. Due to the uncertainties on the external torque on ITER, which is the main source of $\gamma_{\mathrm{ExB}, \mathrm{ext}}$, there is no consensus on the value for this parameter, however it is expected to be considerably lower than in present day tokamaks due to the low torque applied. Therefore, a reduced value, in agreement with ITER integrated modelling simulations [12], has been assumed, $\gamma_{\mathrm{ExB}, \mathrm{ext}}=0.01$. A summary of the heat fluxes together with the 
maximum linear growth rate, $\gamma_{\max }$, and $\mathrm{k}_{\mathrm{y}, \max }$ is shown in table II. Additionally, the influence of the zonal flow activity is evaluated by means of the ratio of the average zonal ExB shear rate, $\gamma_{\text {ExB,zonal, }}$, and $\gamma_{\max }$. The shear rate is defined as $\gamma_{E \times B, \text { zonal }}=\frac{\partial}{\partial r}\left\langle v_{E \times B}\right\rangle$ where $\quad v_{E \times B}$ is the average zonal flow ExB. The values of $\gamma_{\text {ExB,zonal }} / \gamma_{\max }$ are also shown in table II for the different plasma conditions assumed.

From DD to DT the heat flux is reduced by $43 \%$, from $Q_{i, D D}=271 \mathrm{~kW} / \mathrm{m}^{2}$ to $Q_{i, D T}=154 \mathrm{~kW} / \mathrm{m}^{2}$ as shown for cases 1 and 2 , which are close to the one obtained in the original ITER simulation in Ref. [9], $Q_{i}=134 \mathrm{~kW} / \mathrm{m}^{2}$. This trend is indeed not following the Gyro-Bohm scaling. On the other hand, when no electromagnetic or external ExB flow shear effects are included, cases 7 and 8, the heat flux obtained for DT is higher than DD and actually it closely replicates the Gyro-Bohm scaling as $Q_{i, D T} / Q_{i, D D}=1.09 \sqrt{m_{D T} / m_{D D}}$, in agreement with previous studies in electrostatic conditions [13]. In order to identify the physical mechanisms which cause the plasma turbulence to deviate from such scaling (and even reducing the heat flux) the same simulations have been performed by removing the ExB external flow shear, performing electrostatic simulations and removing both effects at the same time. The results with $\gamma_{\mathrm{ExB}, \mathrm{ext}}=0$ (cases 3 and 4) but including electromagnetic effects show that the strong heat flux difference between DD and DT for cases 1 and 2 is now reduced to just $15 \%$ with $Q_{i, D D}=363 \mathrm{~kW} / \mathrm{m}^{2}$ and $Q_{i, D T}=308 \mathrm{~kW} / \mathrm{m}^{2}$, which means that the ExB flow shear is more effective for quenching heat transport at higher mass when $\gamma_{\text {ExB,ext }}$ is constant. When performing electrostatic simulations but still including external ExB flow shear, cases 5 and $6, Q_{i, D D}=1505 \mathrm{~kW} / \mathrm{m}^{2}$ and $Q_{i, D T}=1270 \mathrm{~kW} / \mathrm{m}^{2}$ and therefore the $43 \%$ difference for the cases 1 and 2 reduces to $15.6 \%$.

A simple way to understand the different impact of the ExB flow shear with the mass can be obtained from the consideration that $\gamma_{\mathrm{ExB}}$, is expected to be independent of the mass, $\gamma_{\mathrm{ExB}} E_{r} / L$, with $\mathrm{L}$ a suitable macroscopic length scale, whereas the ITG growth rate scales as $\gamma_{I T G} v_{t h, i} / L$, with $v_{t h, i}=\sqrt{T_{i} / m_{i}}$, and therefore the ratio $\gamma_{E \times B} / \gamma_{\text {ITG }}$, a measure of the impact of the external ExB flow shear on turbulence, scales as $\gamma_{E \times B} / \gamma_{\text {ITG }} m_{i}^{1 / 2}$ indicating that the effectiveness of the ExB flow shear for quenching ITG transport increases with the mass at constant $\gamma_{E \times B}$. However, in realistic isotope experiments, the torque for different isotopes is in general different [14], which may lead to different $\gamma_{\mathrm{ExB}}$ for the different species. The final impact, therefore, would require integrated modelling, something that it is already being developed [14] but requires computational resources beyond the reach at the moment. 
It is worth pointing out that the pfs destabilization also affects DD and DT plasmas in a different way. In cases 9 (DT) and 10 (DD), electrostatic simulations are performed including ExB flow shear but without the correspondent pfs destabilization. Comparing these cases with the full ExB and parallel flow shear effects activated (cases 5 and 6), we observe a strong heat flux increase from $Q_{i, D D}=1303 \mathrm{~kW} / \mathrm{m}^{2}$ for case 10 to $Q_{i, D D}=1505 \mathrm{~kW} / \mathrm{m}^{2}$ for case 6 when the pfs destabilization is included for the DD mixture. Comparing case 9 and 5, however, the heat flux just marginally increases from $Q_{i, D T}=1260 \mathrm{~kW} / \mathrm{m}^{2}$ to $Q_{i, D T}=1270 \mathrm{~kW} / \mathrm{m}^{2}$. These results highlight the important role as well of the $\mathrm{q} / \varepsilon$ factor, with $\varepsilon$ the inverse aspect ratio, which determines the ratio between the parallel and perpendicular components of the magnetic field.

The zonal flow impact has been analyzed by calculating the ratio $\gamma_{\mathrm{ExB}, z \text { onal }} / \gamma_{\max }$. Comparison of the full electromagnetic DT and DD cases (3 and 4) and electrostatic ones (7 and 8), shows that the impact of zonal flow is stronger for DT than DD. However there is no direct translation of this trend to the heat fluxes, as for the cases 7 and 8 the heat flux is higher for DT, in spite of the fact that $\gamma_{\mathrm{ExB}, \mathrm{zonal}} / \gamma_{\max }$ is also higher.
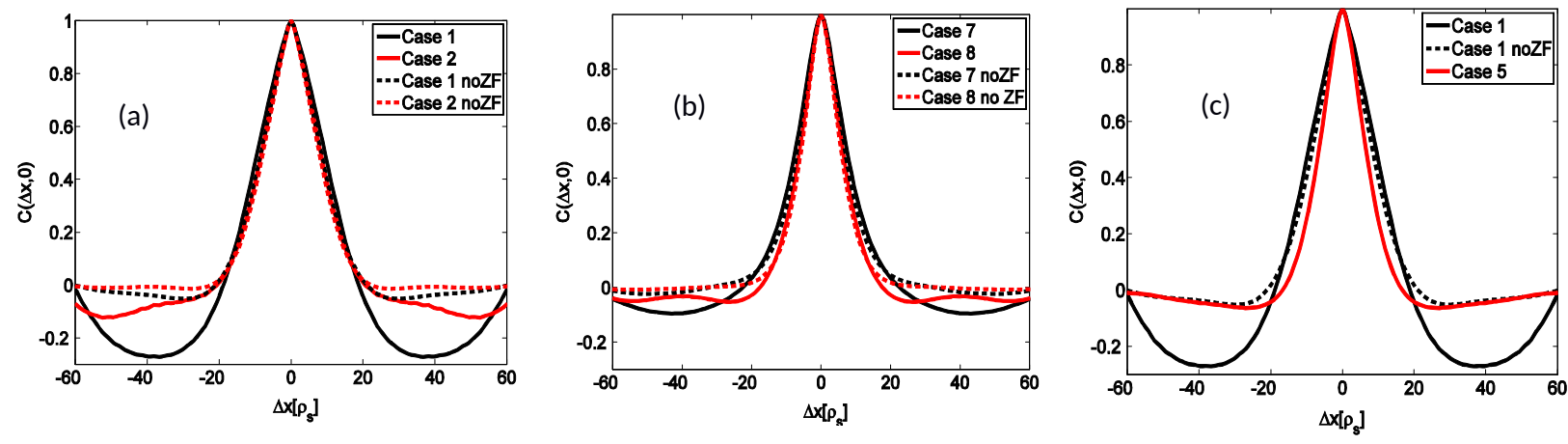

Figure 2 (a) Electrostatic potential equal-time two point correlation function as a function of the radial distance $\Delta x \quad$ in

$\rho_{s} \quad$ units for DT and DD plasma including $\gamma_{\mathrm{ExB}, \mathrm{ext}}$ and electromagnetic effects (cases 1 and 2). The zonal flow component is removed in the post-processing analysis (b) Same analysis excluding $\gamma_{\mathrm{ExB}, \mathrm{ext}}$ and electromagnetic effects (cases 7 and 8). (c) Impact on the electrostatic potential equal-time two point correlation function of the electromagnetic effects in a DT plasma

It is worth to point out that this strong impact of the electromagnetic effects on the isotope effect is a pure non-linear effect as the linear analysis showed that $\gamma_{\max }$ closely follows Gyro-Bohm scaling. Of particular interest is whether the so called quasi-linear models for the heat flux, which usually use the weight $q_{l}=\left|\gamma / k_{y}^{2}\right|_{\max }$, where the maximum is taken over the k-spectrum, as a mixing length rule for the calculation of the heat transport, can reproduce the trend found in this paper in the case of GyroBohm breaking due to electromagnetic effects. As shown in table II, $q_{l}$ is able to capture the reversal of the isotope effect on the heat flux from the cases 7 and 8 to the cases 4 and 5 when the electromagnetic effects are taken into account, however, the magnitude is much weaker than the full 
non-linear simulations. It is worth to clarify that the $\mathrm{k}_{\mathrm{y}}$ corresponding to $\left|\gamma / k_{y}^{2}\right|_{\max }$ is $\mathrm{k}_{\mathrm{y}}=0.35$ for all the cases.

In order to verify the nature of the heat transport when there is a deviation from Gyro-Bohm scaling, the electrostatic potential, $\varnothing$, equal-time two point correlation function has been analyzed with the aim of having a deeper insight on the physical mechanisms playing a role in the isotope effect. This is defined as

$$
C(x, \theta=0, \Delta x)=\frac{\langle\varnothing(x, \theta=0) \varnothing(x+\Delta x, \theta=0)\rangle}{\left(\left\langle\varnothing(x, \theta=0)^{2}\right\rangle\left\langle(x+\Delta x, \theta=0)^{2}\right\rangle\right)^{1 / 2}}(1)
$$

where $\theta$ is the poloidal angle and \langle\rangle the time average calculated over the saturated part of the simulation. The structure of $C(x, \theta=0, \Delta x)$ provides information on both short and long correlation lengths of the turbulence and therefore on their role in the heat transport process. The correlation length $L_{C}$ is defined from the condition $C\left(x, \theta=0, L_{C}\right)=1 / e . L_{C}$ increases following Gyro-Bohm scaling from case 2 with DD, $L_{C}=10.3 \rho_{s}$ up to $L_{C}=11.5 \rho_{s}$ for the case 1 (DT), in spite of the fact that the heat flux reduces with DT. This trend has been recently confirmed in the tokamak ASDEX Upgrade when performing $\rho^{*}$ scaling experiments with hydrogen and deuterium as the main plasma gases [6], indicating a possible common origin for the isotope effect in single and multi-ion plasmas, something that must be confirmed with further analyses. On the other hand, for the cases 7 and 8 (ExB and electromagnetic effects removed), both the heat flux and the correlation length now follow a similar trend from DD, $L_{C}=8.03 \rho_{s}$, to DT, $L_{C}=9.44 \rho_{s}$. This apparent contradiction can be understood by analyzing the full correlation function, i.e. analyzing long range correlations, shown in figure $2 \mathrm{a}$ for both cases, including and excluding the zonal flow component in the post processing analysis. There is a strong anticorrelation region for high $\quad \Delta \quad \mathrm{x}>20 \rho_{\mathrm{s}}$ for case 1 (DT) which is much weaker for case 2 (DD). The origin of this effect is the strong contribution of the zonal flow component for the DT mixture compared to DD. The same comparison is made for cases 7 and 8 when all the effects are suppressed, shown in figure $2 \mathrm{~b}$. The anticorrelation region is very much reduced for both cases although it is still evident that the zonal flow component is stronger for DT. From this analysis it is apparent that in the electrostatic case the stronger zonal flow for DT cannot compensate for the increased correlation length obtained from DD to DT and the general flux follows Gyro-Bohm scaling. The crucial role of electromagnetic effects is even more clearly illustrated in figure 2c, showing that the strong zonal flow activity, associated to the anticorrelation region, is mostly suppressed when the electromagnetic effects are removed. Therefore, the Gyro-Bohm scaling, present at short scale length in any plasma condition, can be counteracted in DT by the long correlation zonal flow activity if the plasma $\beta$ is high enough. The link between electromagnetic effects and stronger zonal flow activity has been studied in several previous studies $[15,16]$, we hope 
that the fact that there is an extra element playing a role, i.e. the mass, may trigger further studies regarding the detailed mechanisms at play.

The previous findings follow the trends found in the experimental DT campaigns performed in TFTR. It was found that the confinement in DT increased with respect to DD with increasing power and in particular, at constant high beta, up to three times less ion heat flux was obtained in the core with DT. The reduction of heat flux obtained in this letter is of that order. However, the physical reasons leading to an isotope effect at different plasma radii than the one assumed here could be different. This possibility will be explored in the future.

In conclusion, the isotope effect identified here can be understood as a complex nonlinear multiscale space interaction involving external ExB flow shear, zonal flow activity, magnetic geometry and electromagnetic effects. The inherent Gyro-Bohm scaling for plasma microturbulence, which exists in any plasma condition and increases the radial correlation length at short scales, is counteracted by the concomitant appearance of these effects, leading to a heat transport of a fundamental mesoscale structure. An optimum plasma configuration for DT operational regimes can be envisaged, favoring high beta and input power with enough torque and fast ion content, characteristic of the so called advanced tokamak regimes. The results obtained in this letter pave the way for future experiments and gyrokinetic simulations. Global electromagnetic gyrokinetic simulations are desirable for a complete understanding of the role of mesoscale physics in the isotope effect. The results obtained here already indicate effects beyond the gyroradius scale but are constrained by the fluxtube simulation domain. Additionally, the full understanding of the isotope effect would also require systematic experimental and simulated numerical scans of ExB flow shear, $\beta$, collisionality and magnetic geometry, in multi and single ion plasmas, and the inclusion of electron scales in order to fully understand the role of electron transport. Due to the substantial amount of computational time required for these simulations this is left for future publications. Moreover, the exact physical mechanism by which there is a coupling between mass, electromagnetic effects and zonal flows is under investigation.

Acknowledgements. The simulations presented in this work were carried out using the HELIOS supercomputer system at Computational Simulation Centre of International Fusion Energy Research Centre (IFERC-CSC), Aomori, Japan.

\section{References}

[1] M. Bessenrodt-Weberpals et al., Nucl. Fusion 33, 1205 (1993).

[2] S.D Scott et al., Phys. Plasmas 2, 6 (1995).

[3] C. Gormezano et al., Phys Rev Lett 805544 (1998).

[4] T. S. Hahm et al., Nucl. Fusion 53, 072002 (2013).

[5] G. Saibene et al., Nucl. Fusion 39, 1133 (1999).

[6] P. Hennequin et al 2015 Plasma physics and control fusion, special issue featuring the invited talks

Proc. 42nd EPS Conf. on Plasma Physics (Lisbon, 22-26 June 2015) p I1.102 
[7] J. Paméla et al., Fusion Engineering Design 87, 590 (2007).

[8] R.V. Budny, J.G. Cordey et al., Nucl. Fusion 56, 056002 (2016).

[9] K. Besseghir et al., Plasma Phys. Control. Fusion 55, 125012 (2013).

[10] F. Jenko, W. Dorland, M. Kotschenreuther and B.N. Rogers, Phys. Plasmas 7, 1904 (2000).

[11] E. A. Belli and J. Candy Phys. Plasmas 17, 112314 (2010).

[12] R. Budny et al., Nucl. Fusion 48075005 (2008).

[13] A. Bustos et al., Phys. Plasmas 22, 012305 (2015).

[14] J. Garcia et al, accepted in ppcf.

[15] M. J. Pueschel et al., Phys. Plasmas 20, 102301 (2013)

[16] J. Anderson et al., Phys. Plasmas 18, 072306 (2011) 